\title{
Investigation and management of Toxoplasma gondii infection in pregnancy and infancy: a prospective study
}

\author{
Paola DI CARLO ${ }^{1}$, Amelia ROMANO ${ }^{2}$, Alessandra CASUCCIO ${ }^{3,}$ *, Salvatore CILLINO ${ }^{3}$, Maria Gabriella SCHIMMENTI ${ }^{2}$, \\ Giorgio MANCUSO ${ }^{4}$, Stella LA CHIUSA ${ }^{4}$, Vincenzo NOVARA ${ }^{2}$, Daniela INGRASSIA ${ }^{1}$, Valentina LI VECCHI ${ }^{1}$, \\ Marcello TRIZZINO ${ }^{1}$, Lucina TITONE ${ }^{1}$ \\ ${ }^{1}$ Department of Sciences for Health Promotion, University of Palermo, Palermo, Italy; ${ }^{2}$ Pediatric Infectious Diseases, Children's Hos- \\ pital "G Di Cristina", ARNAS Civico, Palermo, Italy; ${ }^{3}$ Department of Experimental Biomedicine and Clinical Neuroscience, University of \\ Palermo, Palermo, Italy; ${ }^{4}$ Clinical Pathology Department, Buccheri-La Ferla Hospital, Palermo, Italy
}

\begin{abstract}
Aim: Toxoplasma gondii infection during pregnancy poses a serious risk to the fetus, therefore timely and accurate diagnosis is essential. The aim of this study was to estimate the frequency of congenital infection via evaluating mother's immunological status and the possibility to improving the diagnostic and therapeutic approaches.

Methods: Eighty five mothers with Toxoplasma seroconversion and their offspring were enrolled (among them, 2 spontaneous abortions were documented in the first trimester). Prenatal PCR diagnosis was carried out on 50 patients (60\%), with 7 positive cases (14\%). Morphological ultrasound scanning revealed anomalies in one fetus. Long-term follow-up included general physical examinations, serological status tested using Western blot, neuro-radiological, ophthalmologic and neurologic examinations, psychological and developmental tests, visual evoked potential tests and audiology tests, as well as anti-Toxoplasma treatment regimes.

Results: Fourteen (17\%) of the infants were infected at one-year serological follow-up. Chi-square for linear trend of vertical transmission from the first to the third trimester was significant ( $P=0.009)$. Western blot analysis showed IgM and IgA in half of the infected infants. In 69 uninfected infants, anti-Toxoplasma IgG immunoblot analysis excluded infection within the 3 months in 18 infants (26\%) and in the others within 6 months of life. The most relevant instrumental findings are described.

Conclusion: Western blot analysis may help to evaluate infection within the 6 months of life. The accuracy of ultrasound imaging to determine the brain damage in the fetus and newborns is doubtful, and should be combined with MR imaging. Multistep approaches can improve the timing of postnatal follow-up.
\end{abstract}

Keywords: Toxoplasma gondii; congenital infection; prenatal screening; serological response; ocular prognosis; neurological prognosis; Western blot analysis

Acta Pharmacologica Sinica (2011) 32: 1063-1070; doi: 10.1038/aps.2011.55; published online 11 Jul 2011

\section{Introduction}

Toxoplasma gondii (TG) infection during pregnancy causes severe congenital infection, and may result in in-utero abortion, fetal death, systemic disease or neuro-ophthalmological disorders $^{[1-3]}$.

Despite great progress in clinical and basic scientific research, there are many unresolved issues in congenital toxoplasmosis (CT) that need to be addressed, such as the type and timing of prenatal screening options given the variability in maternal serological response, and their cost-effectiveness ${ }^{[4-6]}$.

\footnotetext{
* To whom correspondence should be addressed.

E-mail casuccio@unipa.it

Received 2011-01-24 Accepted 2011-04-13
}

Recent European prospective cohort studies point out that notwithstanding early maternal and post-natal standard antiparasitic treatment, the most effective approach to preventing Toxoplasma gondii-related injuries remains controversial. Other medical strategies, especially long-term follow-up programs, are recommended to monitor ocular and neurological progno$\operatorname{sis}^{[7-10]}$.

Moreover, in the post-natal period it is crucial to be able to confirm or rule out infection as soon as possible. According to case definition criteria for $\mathrm{CT}$, pediatricians should treat all infants born to mothers with a positive serological diagnosis of primary TG infection until T gondii IgG antibodies disappear ${ }^{[11]}$.

In addition to conventional laboratory methodologies, West- 
ern blot assay has been developed to differentiate between maternal specific antibodies and those synthesized by the newborn infant within the first six months of life ${ }^{[12,13]}$.

The aim of the present study was to estimate frequency of congenital infection based on the mother's immunological status in terms of infection and time of acquisition during pregnancy, and to assess the possibility of modulating the diagnostic and therapeutic approach according to the risk determined for each individual newborn. We report the results of a prospective serological and clinical follow-up program to monitor congenital Toxoplasma gondii infection, conducted at a Sicilian (Italy) paediatric tertiary referral centre after the introduction of Western blot analysis.

\section{Materials and methods}

Study design: 85 mothers with toxoplasmosis seroconversion and their offspring were enrolled in the study from January 2003 to December 2008.

Patients (mothers): Eighty-five HIV negative women who showed seroconversion (change from negative to positive specific IgG antibodies) to Toxoplasma gondii infection during pregnancy were included in the study.

Acute Toxoplasma infection was detected during monthly prenatal screening for toxoplasmosis at the ARNAS Hospital and Buccheri-La Ferla Hospital in Palermo, Italy.

Maternal and pediatric serological and molecular investigations were conducted at the Department of Pathology, Buccheri La Ferla Hospital, Palermo, Italy.

The study protocol was approved by the Ethics Committee of the University of Palermo (Italy) and informed consent was sought in accordance with the principles of the Declaration of Helsinki. Patients were thoroughly informed about the procedures and written informed consent was obtained from each of them.

Prenatal diagnosis: The women had a foetal ultrasound scan every two months during their pregnancy. Once informed consent was granted, amniotic fluid was drawn 4 to 5 weeks after the estimated date of seroconversion and always after 16-18 weeks of amenorrhea. PCR was performed on amniotic fluid to test for Toxoplasma.

Nested PCR was performed from 2003 to 2005 using the B1 gene (GenBank accession number AF179871), following the manufacturer's recommendations (SORIN BIOMEDICASaluggia, Vercelli, Italy $)^{[13]}$. In January 2006, quantitative real time PCR using RE sequence was introduced (GenBank accession number AF146527) (BioQuant $T$ gondii-529, BIORAD iCycler iQ, USA, Biodiversity-Brescia, Italy $)^{[14]}$.

Prenatal treatment: All the women received a standard course of spiramycin (SP) therapy $\left(9 \times 10^{6}\right.$ units $\left./ d\right)$ until their amniocentesis results were available.

If amniocentesis confirmed fetal infection, maternal infection was acquired after 23 weeks or infection was highly suspected (eg because of fetal abnormalities consistent with congenital toxoplasmosis detected by ultrasound examination), treatment consisted of pyrimethamine (PYR $50 \mathrm{mg} / \mathrm{d}$ ) and sulphadiazine (SDX $3 \mathrm{~g} / \mathrm{d})$ until delivery.
Patients (infants): Congenital infection was defined by the persistence of specific IgG antibodies beyond 12 months of age, with or without clinical signs. From birth, long term follow-up of the infants included general physical examinations, serological status, neuro-radiological findings, ophthalmologic and neurologic examinations, psychological and developmental tests, visual evoked potential tests (VEPs) and audiology testing (using BAEPs and impedance audiometry).

All eye examinations were carried out by an experienced ophthalmologist, who recorded findings on a standardized form. Visual acuity and abnormalities relating to the anterior and posterior segments of both eyes after pupillary dilatation were considered, using direct ophthalmoscopy, Schepens binocular indirect ophthalmoscopy and/or no-contact widefield lenses (90 diopter) for slit lamp, depending on the child's age and compliance. Under 3 years of age, visual acuity was assessed using Parinaud charts and was considered as normal when $>2$. Snellen charts were used for older children, and visual acuity was considered as normal when $>20 / 25$. The children were monitored for VEPs and BAEPs until 3 years of age.

Infants underwent brain ultrasound (US) examinations via the bregmatic fontanelle until six months of age (Aloke SSD650 equipped with a 5-8 MHz sector transducer). When necessary, also a CT scan (scanning at 3-mm collimation with 5 -mm interval and at 10-mm collimation) or MRI (1.6 to 4 $\mathrm{mm}$ slice thickness, sequence T1-W conventional spin-echo or T2-W fast spin-echo) of the head was performed.

Serological investigation: Serological analysis was carried out with well-established conventional serological methods. Congenital toxoplasmosis was diagnosed by determining the presence of Toxoplasma-specific IgM, IgA and IgG antibodies at birth, within the first month of life and at 3 months, and of Toxoplasma-specific IgG also at 6, 9, and 12 months of age [ETI TOXOK-M reverse, ETI TOXOK-G PLUS (DiaSorin, Saluggia, Italy), Toxo-IgM ISAGA, Toxo-IgG VIDAS, Toxo-IgG avidity VIDAS (BioMerieux, Marcy-l'Etoile, France)]. Consistent with NCCLS recommendations, the kits showed sensitivity and specificity of $>95$ and $>98 \%-99 \%$ respectively. All patients were double-tested, with an intra-assay coefficient of variation (CV) of $<2 \%$.

Comparative immunological profile analysis of maternal serum samples, cord blood, neonatal sera and the infants' follow-up sera was performed with Toxoplasma IgG, IgM and IgA Western blot (WB), as well as conventional immunoenzymatic serological tests ${ }^{[13]}$. WB analysis used Toxoplasma gondii RH strain tachyzoite protein lysates and allowed us to identify antigens of 16, 27, 30 (p30), 35, 40, 60, 67, 70, 88, 94, and 110$\mathrm{kDa}$ bands. The test was performed following the manufacturer's instructions (GenID GmbH, Strassberg, Germany), using the AID SCAN System.

Western blot was considered positive when at least two specific immunoglobulin (IgG) bands were clearly present in the infant sample but not in the mother's serum. IgM WB was performed on sera collected at birth or within $14 \mathrm{~d}$ after birth, and at 2 and 3 months of life, whereas IgG WB was continued 
to 6 months of life.

Post-natal treatment: Different anti-Toxoplasma treatment regimes were initiated according to prenatal diagnosis, as follows:

Neonates born to mothers whose amniocentesis result was positive for Toxoplasma were treated with a combination of PYR (3 mg/kg every $3 \mathrm{~d}$ ) and sulfadiazine (SDX) $(25 \mathrm{mg} / \mathrm{kg}$ every $8 \mathrm{~h}$ ), with $100 \mathrm{mg} / \mathrm{kg}$ of folinic acid daily for 4 weeks, followed by SP $\left(0.375 \times 10^{6} \mathrm{U} / \mathrm{kg}\right.$ daily $)$ for 4 weeks. Their hematologic and renal status was monitored weekly until one year of age.

Neonates born to mothers with negative amniocentesis were given spiramycin until specific antibodies disappeared or WB analysis was negative.

Neonates born to mothers who did not have an amniocentesis were given a combination of PYR-SDX and SP as reported in paragraph 1, and hematologic and renal function was monitored weekly until serological follow-up showed that specific IgG had disappeared or IgG WB analysis was negative.

Compliance with treatment was assessed in interviews with parents; periodic blood tests (chemical evaluation) were done to identify possible side effects.

\section{Statistical analysis}

Demographic and clinical data were collected and analyzed using SPSS Software, version 14.0 (SPSS, Inc, Chicago, Ill, US). Statistical analysis of quantitative data, including descriptive statistics, was performed for all the items. The chi square test for linear trend was performed to evaluate vertical transmission of infection through the pregnancy trimesters. All $P$ values were two-sided and $P$ values less than 0.05 were considered to indicate statistical significance.

\section{Results}

At the final examination, the children were aged between 2 and 6 years (median age was 4.8 years), and had been followed up for at least 2 years (median 4.6 years; range 2-6 years).

Two pregnancies terminated in the first trimester, with fetal loss before amniocentesis. A total of 83 infants born to 85 mothers were included in the study and none of the mothers or the children were immunocompromised. Time of seroconversion was dated in the first trimester for 38 of the 85 pregnant women (45\%), in the second trimester for $24(28 \%)$ and in the third trimester for the remaining 23 (27\%) .

Prenatal diagnosis by PCR testing on amniotic fluid was attempted for 50 of the 83 women who went on to give birth $(60 \%)$, with positive results in seven cases $(14 \%)$; the other women either did not consent to having the test $(10,12 \%)$ or seroconverted late in the third trimester $(23,28 \%)$. All the mothers with positive PCR results chose to continue their pregnancy, and prenatal ultrasound screening, performed every trimester on all of the patients, detected ventriculomegaly in one woman with a fetus at 31-weeks' gestation.

Fourteen of the 83 infants in the study (17\%) were congenitally infected with Toxoplasma gondii and 69 (83\%) proved to be uninfected. Chi-square test for linear trend of vertical transmission from the first to the third trimester [2/38 in the $1^{\text {st }}$ trimester $(\mathrm{OR}=1.00)$; 5/24 in the second $(\mathrm{OR}=4.74)$; and $7 / 23$ in the third trimester $(\mathrm{OR}=7.87)]$ was significant $(P=0.009)$.

Six of the 14 infected children (43\%) were asymptomatic at birth, and clinical and instrumental follow-up was negative, whereas the other 8 (57\%) showed features of TG infection (Table 1). This group included all fetuses with positive prenatal PCR results; four of them showed symptoms of TG infection at birth and 6 were small for gestational age (SGA). Four children $(28.6 \%)$ had cerebral lesions and ocular fundus pathologies 3 cases with foci of peripheral chorioretinitis and one with peripheral chorioretinitis in the left eye (LE) and macular chorioretinitis in the right eye (RE)]. Visual outcome was good in all cases, with normal visual acuity and VEPs within the normal range.

Seven of the TG-infected infants had received a positive prenatal diagnosis, whereas the mothers of the remaining infants either did not consent ( $1 \mathrm{pt})$ or seroconverted late in the third trimester (6 pts).

In accordance with guidelines for anti-parasitic treatment, all the infected newborns were treated with a combination of PYR-SDX and SP. Compliance to therapy was assessed by interviews with parents, and was good in all cases.

The serological investigation using WB showed IgM and

Table 1. Clinical and investigation findings during the pre- and post-natal era in eight symptomatic newborns with congenital $T$ gondii infection.

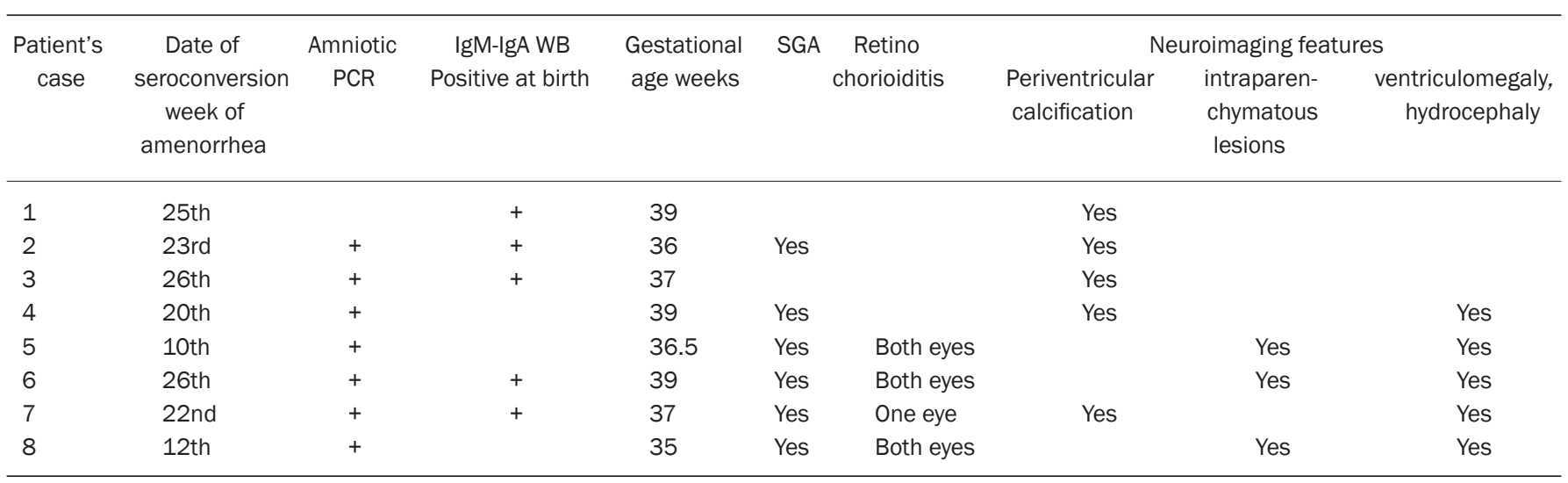


IgA in $7(50 \%)$ of the 14 infected patients, whereas IgM ISAGA combined with $\operatorname{IgA}$ reacted positive in 4 of them $(28.6 \%)$ (Table 2). Most of these neonates had been infected after 21 weeks of gestation (Table 1). In the absence of IgM, comparative anti Toxoplasma IgG immunoblot analysis revealed different mother-child IgG profiles in the first trimester of life in 3 infected infants, whereas in the others the interpretation became clear within six months of life.

Table 2. Complementary detection of 83 enrolled infants' Toxoplasmaspecific antibodies by different techniques during one year of follow-up.

\begin{tabular}{|c|c|c|c|c|c|c|}
\hline \multirow{2}{*}{ Patients } & \multirow{2}{*}{ Methods } & \multicolumn{5}{|c|}{ Detection at following times after birth } \\
\hline & & $0-30 d$ & 3 month & 6 month & 9 month & 1 year \\
\hline \multirow[t]{4}{*}{$\begin{array}{l}\text { Infected } \\
(14)\end{array}$} & $\begin{array}{l}\text { IgM-ISAGA+IgA } \\
\text { Positive }\end{array}$ & 4 & & & & \\
\hline & IgM-IgA WB & 7 & & & & \\
\hline & Positive & & & & & \\
\hline & $\begin{array}{l}\text { IgG-Western blot } \\
\text { Positive }\end{array}$ & 7 & 3 & 14 & & \\
\hline \multirow[t]{2}{*}{$\begin{array}{l}\text { Uninfected } \\
\text { (69) }\end{array}$} & $\begin{array}{l}\text { IgG-ELISA } \\
\text { Positive }\end{array}$ & 69 & 55 & 26 & 3 & 0 \\
\hline & $\begin{array}{l}\text { IgG-Western blot } \\
\text { Negative }\end{array}$ & 1 & 17 & 69 & & \\
\hline
\end{tabular}

In infants uninfected at birth (69), comparative anti Toxoplasma IgG immunoblot analysis permitted us to exclude infection within the first three months of life in 18 children $(26 \%)$ and in the others within six months of life, whereas the conventional immunoenzymatic serological test showed specific IgG persistence at six months of life in about one third of the infants, and in 3 cases at nine months of life.

The instrumental follow-up showed typical periventricular calcification in three cases, whereas relevant involvement of the brain was observed in five infants. In the following section we report these significant radiological findings.

In case 4, clinical neurological examination was normal and ultrasound follow-up of the brain showed intracranial calcification in the lateral ventricles and in the frontal and parietal lobes (Figure 1A). A magnetic resonance (MR) performed at 9
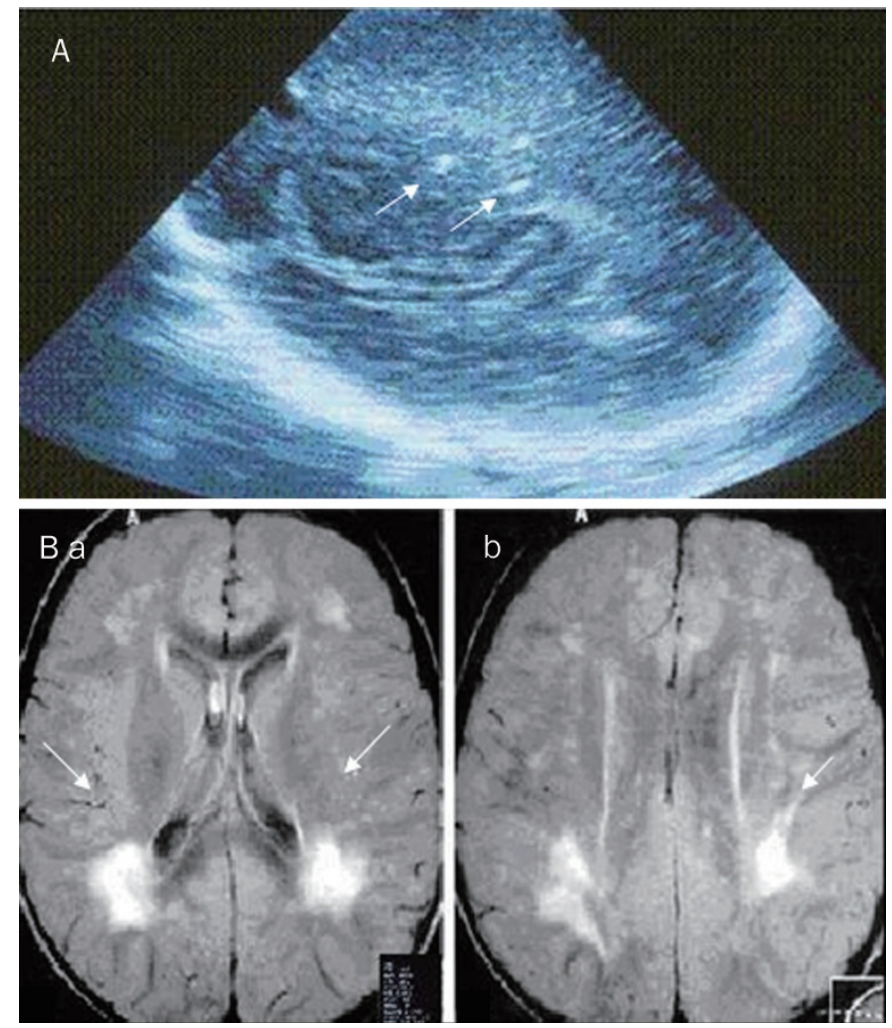

Figure 1. (A) Coronal US image using a vector scan showing brain calcification in lateral ventricle (arrows). (B) Patient 1. (a) MRI images showing focal lesions on the right side and (b) another smaller lesion in the subcortical posterior parietal area (arrows).

months of age showed intra-parenchymal brain lesions (Figure 1B). At present, the patient is in his fourth year of follow-up and his psychomotor development is normal.

In case 5, an ultrasound scan performed at 1 month of age showed a hypoechogenic area in the paraventricular white matter (Figure 2A), and at 2 months of age, MRI imaging showed encephalitic foci and cortical brain atrophy and ventricular dilation (Figure 2B). An ophthalmoscopic examination revealed peripheral bilateral inactive chorioretinitis foci with no vitreous inflammation. Tone and motor abnormalities resolved by 1 year of age, and after five years of follow-up, the
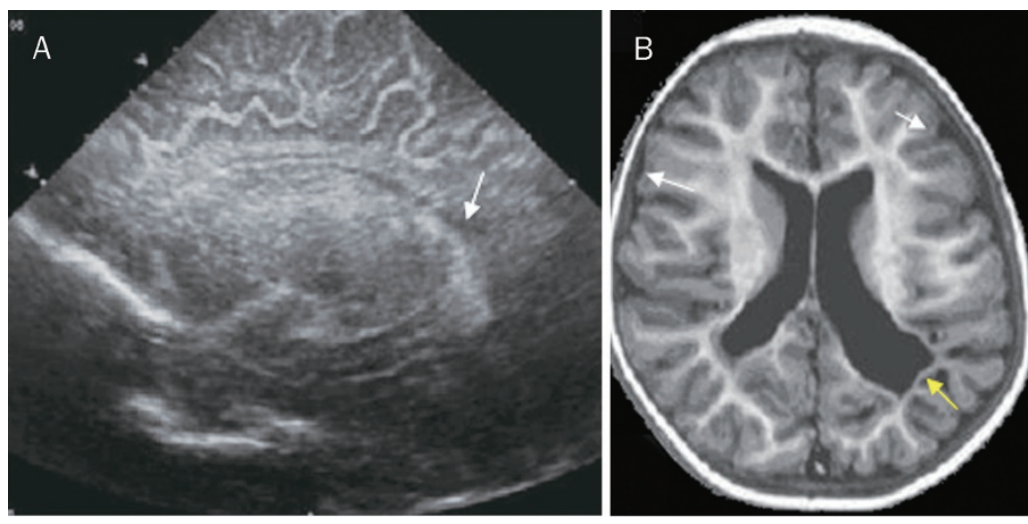

Figure 2. (A) Cranial ultrasound parasagittal views showing hypoechogenic area in the left paraventricular white matter (arrow). (B) T2 MR image showing encephalitic foci and cortical brain atrophy in the parietal area, white matter atrophy (white arrows), ventricular dilation, irregular angulated ventricles (yellow arrow). 
child's psychomotor development is within normal limits.

In case 6, ventriculomegaly was detected during prenatal US follow-up. After birth, the features were confirmed by brain US and MR imaging, which showed lateral ventricular enlargement and obstructive hydrocephalus with aqueductal stenosis (Figure 3A, B). A ventriculotomy was necessary to treat the hydrocephalus, and a subsequent MR examination performed 6 months later revealed complete resolution (Figure 3C). An eye examination showed peripheral chorioretinitis without any surrounding activity. Seizures and motor abnormalities were resolved during therapy. The patient has been regularly attending our Day Hospital Service for three years and his psychomotor development is normal.

In case 7 , ophthalmoscopy at birth revealed a peripheral inactive chorioretinitis scar. Ultrasound evaluation performed after delivery showed severe tetraventricular hydrocephalus and calcifications scattered around lateral ventricles (Figure 4). An MRI scan performed at 6 months of age revealed a reduction of hydrocephalus.

In case 8 , pregnancy had been uneventful, although a detailed morphological ultrasound scan at 18 weeks had revealed biparietal measurement $(30 \mathrm{~mm})$ and head circumference $(110 \mathrm{~mm})$ below the 5 th percentile, corresponding to 16 weeks gestation; this finding disappeared in all subsequent examinations. At birth, a visual evaluation showed microphthalmia, LE peripheral and RE macular chorioretinitis. Ultrasonography showed enlarged lateral ventricles and findings suggestive of aqueductal stenosis (Figure 5A). A comparative MRI investigation confirmed ventricular abnormalities

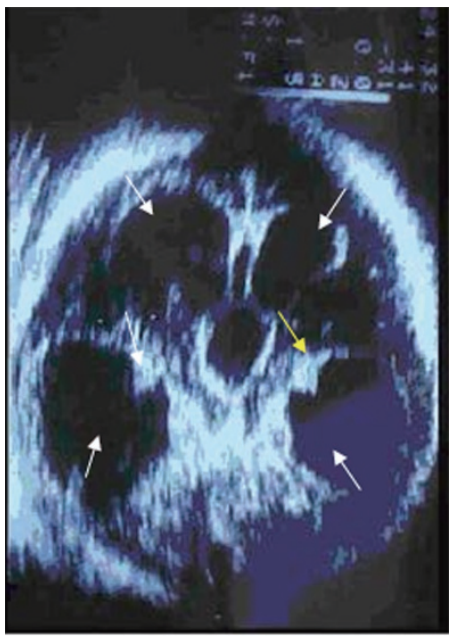

Figure 4. Axial US image using a vector transducer, showing severe tetraventricular hydrocephalus (white arrows), calcifications scattered around lateral ventricles (yellow arrow).

detected by US and moreover showed brain-damage (Figure 5B). Motor abnormalities accompanied by behaviour disturbances and seizures persisted after one year of follow-up.

\section{Discussion}

The prevalence of congenital toxoplasmosis differs from one country to another. Various European works have focused on the cost-benefit ratio of wide-scale screening to prevent infec-
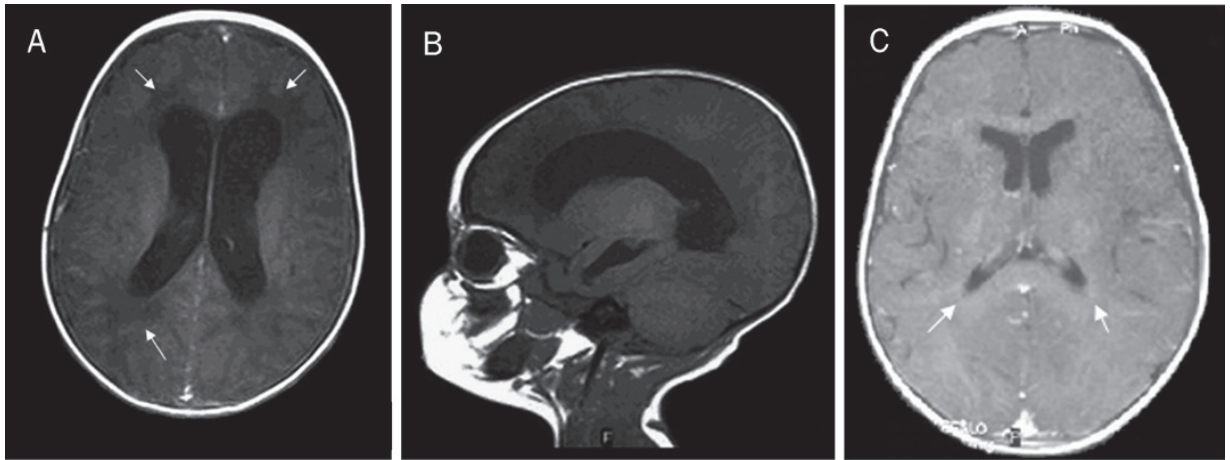

Figure 3. (A, B) Axial MR image showing evident hydrocephalic enlargement of lateral ventricles, area of hypointensity in the periventricular white matter to be attributed to ependymal transudation (arrows). (C) MR image showing a resolution of previous features (arrows).
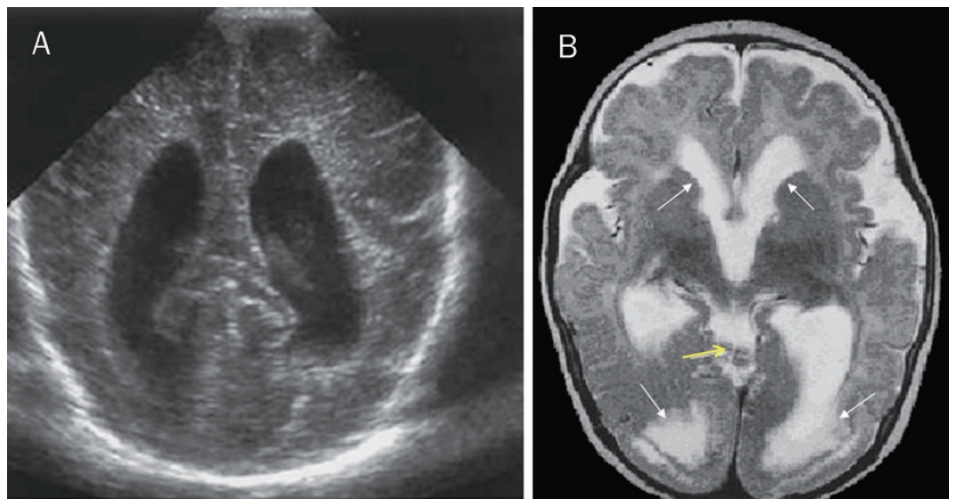

Figure 5. (A) Cranial ultrasound shows enlarged lateral ventricles. (B) Axial images show triventricular hydrocephalus, cyst-like inclusions (yellow arrow), cortical dysplasia, occipital polymicrogyria, white matter hyperintensity (transependymal transudation localized in the posterior horn of lateral ventricles) (white arrows). 
tion, despite the fact that the consequences of the disease can be very disabling in childhood and adolescence and, above all, require considerations of an ethical nature regarding the pregnant woman and the well-being of the unborn child ${ }^{[8,15]}$.

Among the poorest populations living in any region like Southern Italy, parasitic diseases such as leshmaniasis and toxoplasmosis remain prevalent ${ }^{[16]}$, and although our small sample size does not permit us to make any consistent conclusions about anti-parasitic treatment, the high risk for pregnant women of transmitting infection to their fetus encourages us to make a number of suggestions.

Our study collected data on a large group of children (36 cases) who were infected in utero by $T$ gondii during the first trimester of pregnancy and whose ultrasound findings remained normal. As reported by Berrebi, our follow-up confirms that their outcome does not differ significantly from that of children infected during the second and third trimester ${ }^{[17]}$.

As regards the prenatal phase, we observed a lower motherto-child transmission rate than in other reports, and concur with a recent meta analysis by the SYROCOT group which found weak evidence that treatment initiated within 3 weeks of seroconversion could reduce the risk of transmission ${ }^{[18]}$.

On the other hand, precocious maternal treatment did not prevent severe neurological and ocular disease, especially in children in whom parasitic infection was confirmed by amniotic PCR results ${ }^{[19]}$. When available, molecular investigations to detect Toxoplasma gondii in amniotic fluid provide useful information for patients and caregivers that can guide decision making and surveillance ${ }^{[14,20]}$. The authors emphasize that all the infants with neuro-ophthalmological specific disease had received a positive prenatal diagnosis, whereas only in one late case of infection did monthly foetal ultrasound investigations give us any information on signs of congenital infection. However, the authors agree on the need for repeated ultrasound examinations, especially in delayed mother-tochild transmission of the disease when amniocentesis is rarely offered $^{[21,22]}$.

Although ultrasound investigation via the bregmatic fontanelle is one of the techniques most frequently used by our staff in the postnatal follow-up, we prefer MRI in congenital toxoplasmosis as it offers more accurate pictures for the diagnosis and follow-up of white matter lesions in the infants, and provides excellent anatomic information on abnormalities in brain parenchyma. Fast magnetic resonance imaging (MRI) is being increasingly used to assess pregnancy management in the event of fetal central nervous system (CNS) anomalies ${ }^{[23]}$.

In the neonatal phase of the study, all the infected infants were put on early treatment with PYR-SDX to one year of age and, for five of them with severe damage, until 18 months of life. Furthermore, we chose to also treat infants born to mothers who did not undergo amniocentesis with PYS-SDX.

Although the authors acknowledge that evidence is lacking on the effectiveness of early postnatal treatment in reducing the risk of subsequent specific lesions, our post-natal followup revealed an improvement in cerebral and ocular lesions. Moreover, some cohort studies have shown that treating an infected neonate early and effectively may lead to an improvement in ocular lesions and reduce the risk of developing ocular disease in the years after birth ${ }^{[24,25]}$. Certainly a multidisciplinary early intervention strategy in the post-natal period can positively influence the cognitive functions of children with visual impairment.

In this case, it is vital that the clinician informs the parents accordingly and motivates their adherence to a long-term follow-up.

Western blot analysis has improved the timing of toxoplasmosis diagnosis in post natal follow-up to about six months of life. In some cases the situation is clear before then, but our own experience suggests that it is advisable not to anticipate diagnosis as the presence of maternal antibodies strongly inhibits the neonate's immunological response ${ }^{[26,27]}$.

It is generally accepted that maternal antibodies can block the offspring's immune response, making it particularly difficult to identify neonatal immunoglobulin soon after birth ${ }^{[1,2,20]}$. Moreover, immunological markers can vary depending on the trimester of infection, and also maternal and neonatal therapeutic treatment received during pregnancy can block or retard the neonate's immune response ${ }^{[26,27]}$. Therefore, even if comparison of maternal and neonatal IgG by immunoblot does not show neonatal immune response to any Toxoplasma gondii antigens in the first trimester of life, this does not unequivocally exclude congenital infection.

In fact, recent studies which have evaluated B-cell subsets and their functional development have shown that memory B-cells are very low at birth and increase significantly at 6 months of life $\mathrm{e}^{[28]}$.

WB analysis is useful in managing children whose mother did not have amniocentesis, and in knowing with greater certainty when to stop therapy in children whose mother had a negative amniocentesis result. In about one third of cases, this reduces the costs associated with treatment and follow-up. It is true that in most cases the clinical picture is already clear by nine months of age, even with conventional methods, and that WB can anticipate the time of diagnosis by about two to three months.

A previous overview of investigation strategies has provided vital information to determine what is the most suitable multi-step laboratory investigation strategy for appropriate and timely clinical management of the infection ${ }^{[29,30]}$.

WB analysis should be incorporated in these TG infection investigation programs and performed in reference centers for the diagnosis of Toxoplasmosis in pregnancy and children. In particular, the authors suggest that WB analysis is useful both in pregnancy, as part of a panel of tests to determine if the mother has an acute Toxoplasma gondii infection, in which case she then has to undergo specific treatment and amniocentesis, and also after birth, when WB results may help pediatricians to clarify any pitfalls that may be encountered during the interpretative process of investigations, especially in the first few months of life.

Toxoplasma gondii is a parasite within the protozoan phylum Apicomplexa that contains pathogens of considerable medical 
and veterinary importance, such as Plasmodium, Toxoplasma, Cryptosporidium, Eimeria, Neospora and Theileria species ${ }^{[31]}$. The apicomplexan parasites Cryptosporidium spp and Neospora spp are epidemiologically relevant for humans; in particular, it is well-known that $C$ parvum is responsible for clinical manifestations in immunodeficient patients. $N$ caninum does not infect humans, although there is some evidence that it might occur as antibodies to $N$ caninum have also been detected in human samples $^{[32,33]}$. Because there are some similarities in molecular composition between both of these parasites and Toxoplasma gondii, the use of whole tachyzoites or tachyzoite-derived antigens in standard serological investigations may result in false positives due to a cross-reaction ${ }^{[34]}$. Although variations in the protein expression profiles of tachyzoites and bradyzoites of $N$ caninum may be identified by Western blotting ${ }^{[35]}$, a cross-reactivity cannot be excluded, and combined molecular diagnostic assays must be applied to confirm Toxoplasma gondii etiology ${ }^{[36,37]}$. Thus, it is important to identify a readilyavailable serological test that is highly sensitive and specific in detecting the infection as early as possible.

In conclusion, clinical recognition and effective management of congenital toxoplasmosis is challenging and the strategy to reduce damages needs a multistep approach in the pre- rather than post-natal era to reduce the high costs of multidisciplinary follow-up.

\section{Author contribution}

Paola DI CARLO, Amelia ROMANO and Lucina TITONE designed and organized the study; Paola DI CARLO, Salvatore CILLINO and Giorgio MANCUSO performed clinical and serological follow-up; Alessandra CASUCCIO analyzed the data and vouch for the analysis results; Maria Gabriella SCHIMMENTI, Stella LA CHIUSA, Valentina LI VECCHI, Daniela INGRASSIA, Vincenzo NOVARA, and Marcello TRIZZINO contributed to collecting data and to enrolling patients at the participating centres. All the authors contributed to writing the paper.

\section{References}

1 Montoya JG, Liesenfeld O. Toxoplasmosis. Lancet 2004; 363: 196576.

2 Remington JS, McLeod R, Thulliez P, Desmonts G. Toxoplasmosis. In: Infectious Diseases of the Fetus and Newborn Infant, 6th edn. Editors: Remington JS, Klein JO, Wilson CB, Baker CJ. Philadelphia, PA: Elsevier-Saunders; 2006. p948-1091.

3 Embleton. The Northern Region's Perinatal Mortality Survey. Fetal and neonatal death from maternally acquired infection. Paediatr Perinat Epidemiol 2001; 15: 54-60.

4 Sterkers Y, Varlet-Marie E, Marty P, Bastien P, on behalf of the ANOFEL Toxoplasma-PCR Quality Control Group. Diversity and evolution of methods and practices for the molecular diagnosis of congenital toxoplasmosis in France: a four years survey. Clin Microbiol Infect Clin Microbiol Infect 2010; 16: 1594-602

5 Maudry A, Chene G, Chatelain R, Patural H, Bellete B, Tisseur B, et al. Bicentric evaluation of six anti-toxoplasma immunoglobulin $\mathrm{G}$ (IgG) automated immunoassays and comparison to the Toxo II IgG Western blot. Clin Vaccine Immunol 2009; 16: 1322-6.
6 Lago EG, Neto EC, Melamed J, Rucks AP, Presotto C, Coelho JC, et al. Congenital toxoplasmosis: late pregnancy infections detected by neonatal screening and maternal serological testing at delivery. Paediatr Perinat Epidemiol 2007; 21: 525-31.

7 Bénard A, Petersen E, Salamon R, Chêne G, Gilbert R, Salmi LR, et al. Survey of European programmes for the epidemiological surveillance of congenital toxoplasmosis. Euro Surveill 2008; 13. pii: 18834.

8 Röser D, Nielsen HV, Petersen E, Saugmann-Jensen P, NørgaardPedersen PB. Congenital toxoplasmosis-a report on the Danish neonatal screening programme 1999-2007. J Inherit Metab Dis 2010; 33: S241-247.

9 Berrébi A, Assouline $\mathrm{C}$, Bessières $\mathrm{MH}$, Lathière $\mathrm{M}$, Cassaing $\mathrm{S}$, Minville $\mathrm{V}$, et al. Long-term outcome of children with congenital toxoplasmosis. Am J Obstet Gynecol 2010; 203: 552.e1-6.

10 Wallon M, Kodjikian L, Binquet C, Garweg J, Fleury J, Quantin C, et al. Long-term ocular prognosis in 327 children with congenital toxoplasmosis. Pediatrics 2004; 113: 1567-72.

11 Lebech M, Joynson DH, Seitz HM, Thulliez P, Gilbert RE, Dutton GN, et al. Classification system and case definitions of Toxoplasma gondii infection in immunocompetent pregnant women and their congenitally infected offspring. European Research Network on Congenital Toxoplasmosis. Eur J Clin Microbiol Infect Dis 1996; 15: 799-805.

12 Tridapalli E, Capretti M, Farneti G, Marangoni A, Cevenini R, Faldella G. Congenital toxoplasmosis: the importance of the Western blot method to avoid unnecessary therapy in potentially infected newborns. Acta Paediatr 2008; 97: 1298-300.

13 Di Carlo P, Casuccio A, La Chiusa S, Mazzola A, Pampinella D, Romano $A$, et al. Diagnosis of congenital toxoplasmosis: pre- and post-natal evaluation in Sicilian (Italy) epidemiological area. Preliminary data. Parassitologia 2007; 49: 39-41.

14 Cassaing $\mathrm{S}$, Bessières MH, Berry A, Berrebi A, Fabre R, Magnaval JF. Comparison between two amplification sets for molecular diagnosis of toxoplasmosis by real-time PCR. J Clin Microbiol 2006; 44: 720-4.

15 Cortina-Borja M, Tan HK, Wallon M, Paul M, Prusa A, Buffolano W, et al. Prenatal treatment for serious neurological sequelae of congenital toxoplasmosis: an observational prospective cohort study. PLoS Med 2010; 7. pii: e1000351.

16 Vesco G, Buffolano W, La Chiusa S, Mancuso G, Caracappa S, Chianca A, et al. Toxoplasma gondii infections in sheep in Sicily, southern Italy. Vet Parasitol 2007; 146: 3-8.

17 Berrebi A, Bardou M, Bessieres MH, Nowakowska D, Castagno R, Rolland $\mathrm{M}$, et al. Outcome for children infected with congenital toxoplasmosis in the first trimester and with normal ultrasound findings: a study of 36 cases. Eur J Obstet Gynecol Reprod Biol 2007; 135: $53-7$

18 SYROCOT (Systematic Review on Congenital Toxoplasmosis) study group, Thiebaut R, Leproust S, Chene G, Gilbert R. Effectiveness of prenatal treatment for congenital toxoplasmosis: a meta-analysis of individual patients' data. Lancet 2007; 369: 115-22.

19 Garcia-Méric P, Franck J, Dumon H, Piarroux R. Management of congenital toxoplasmosis in France: current data. Presse Med 2010; 39: $530-8$.

20 Montoya JG, Remington JS. Management of Toxoplasma gondii infection during pregnancy. Clin Infect Dis 2008; 47: 554-66.

21 Gay-Andrieu F, Marty P, Pialat J, Sournies G, Drier de Laforte T, Peyron F. Fetal toxoplasmosis and negative amniocentesis: necessity of an ultrasound follow-up. Prenat Diagn 2003; 23: 558-60.

22 Villena I, Bory JP, Chemla C, Hornoy P, Pinon JM. Congenital toxoplasmosis: necessity of clinical and ultrasound follow-up despite negative amniocentesis. Prenat Diagn 2003; 23: 1098-9.

23 Herman-Sucharska I, Bekiesińska-Figatowska M, Urbanik A. Fetal 
central nervous system malformations on MR images. Brain Dev 2009; 31: 185-99.

24 Freeman K, Tan HK, Prusa A, Petersen E, Buffolano W, Malm G, et al. European Multicentre Study on Congenital Toxoplasmosis. Predictors of retinochoroiditis in children with congenital toxoplasmosis: European, prospective cohort study. Pediatrics 2008; 121: e1215-22.

25 Roizen N, Kasza K, Karrison T, Mets M, Noble AG, Boyer K, et al. Impact of visual impairment on measures of cognitive function for children with congenital toxoplasmosis: implications for compensatory intervention strategies. Pediatrics 2006; 118: e379-90.

26 Bessières M H, Berrebi A, Rolland M, Bloom MC, Roques C, Cassaing $\mathrm{S}$, et al. Neonatal screening for congenital toxoplasmosis in a cohort of 165 women infected during pregnancy and influence of in utero treatment on the results of neonatal tests. Eur J Obstet Gynecol Reprod Biol 2001; 94: 37-45.

27 Meroni V, Genco F, Tinelli C, Lanzarini P, Bollani L, Stronati M, et al. Spiramycin treatment of Toxoplasma gondii infection in pregnant women impairs the production and the avidity maturation of $T$ gondiispecific immunoglobulin $\mathrm{G}$ antibodies. Clin Vaccine Immunol 2009; 16: $1517-20$.

28 Avanzini MA, Maccario R, Belloni C, Carrera G, Bertaina A, Cagliuso M, et al. B lymphocyte subsets and their functional activity in the early months of life. Int J Immunopathol Pharmacol 2010; 23: 247-54.

29 Pinon JM, Dumon H, Chemla C, Franck J, Petersen E, Lebech M, et al. Strategy for diagnosis of congenital toxoplasmosis: evaluation of methods comparing mothers and newborns and standard methods for postnatal detection of immunoglobulin G, M, and A antibodies. J Clin Microbiol 2001; 39: 2267-71.
30 Sensini A. Toxoplasma gondii infection in pregnancy: opportunities and pitfalls of serological diagnosis. Clin Microbiol Infect 2006; 12 : 504-12.

31 Kim K, Weiss LM. Toxoplasma gondii: the model apicomplexan. Int J Parasitol 2004; 34: 423-32.

32 Barratt JL, Harkness J, Marriott D, Ellis JT, Stark D. Importance of nonenteric protozoan infections in immunocompromised people. Clin Microbiol Rev 2010; 23: 795-836.

33 Ibrahim HM, Huang P, Salem TA, Talaat RM, Nasr MI, Xuan X, et al. Short report: prevalence of Neospora caninum and Toxoplasma gondii antibodies in northern Egypt. Am J Trop Med Hyg 2009; 80: 263-7.

34 Chahan B, Gaturaga I, Huang X, Liao M, Fukumoto S, Hirata H, et al. Serodiagnosis of Neospra caninum infection in cattle by enzymelinked immunosorbent assay with recombinant truncated NcSAG1. Vet Parasitol 2003; 118: 177-85.

35 Kang SW, Lee EH, Jean YH, Choe SE, Van Quyen D, Lee MS. The differential protein expression profiles and immunogenicity of tachyzoites and bradyzoites of in vitro cultured Neospora caninum. Parasitol Res 2008; 103: 905-13.

36 Wang CR, Zhai YQ, Zhao XC, Tan QJ, Chen J, Chen AH, et al. Preliminary application of PCR-based assay for the detection of Neospora caninum in bovine aborted fetus. Zhongguo Ji Sheng Chong Xue Yu Ji Sheng Chong Bing Za Zhi 2009; 27: 140-3.

37 Edelhofer R, Loeschenberger K, Peschke R, Sager H, Nowotny N, Kolodziejek J, et al. First PCR-confirmed report of a Neospora caninum-associated bovine abortion in Austria. Vet Rec 2003; 152: 471-3. 